\title{
Revealing the lifelong bio-persistent crystal structure of an asbestos fibre
} C. Giacobbe ${ }^{a}$, Dario Di Giuseppe ${ }^{\text {bc }}$, Alessandro Zoboli ${ }^{b}$, Paola Bonasoni ${ }^{d}$, Anna Moliternie, Jonathan Wright ${ }^{a}$ and Alessandro F. Gualtierib

${ }^{a}$ European Synchrotron Radiation Facility, 71, Avenue Des Martyrs, Grenoble, 38040, France. ${ }^{b}$ Department of Chemical and Geological Sciences, University of Modena and Reggio Emilia, Modena, 41121, Italy. ${ }^{c}$ Department of Sciences and Methods for Engineering, University of Modena and Reggio Emilia, Via Amendola, 2, Reggio Emilia, 42122, Italy. ${ }^{d}$ Pathology Unit, Azienda Unità Sanitaria Locale - IRCCS, Reggio Emilia, Italy. ${ }^{e}$ Istituto di Cristallografia, CNR, Via Amendola, 122/o, Bari, 70126, Italy

carlotta.giacobbe@esrf.fr

Humans have used asbestos for about 5,000 years in various parts of the world [1] because of its outstanding properties. The massive use of asbestos turned out to be a global environmental problem when animal carcinogenicity tests and longterm epidemiological studies proved that inhalation of asbestos fibres may induce fatal lung diseases like asbestosis, carcinoma, malignant mesothelioma (MM) and many more after a latency period of decades [2].

Besides the morphological and chemical characterization conveyed by ESD-supported electron microscopy, X-ray diffraction is considered a reliable tool for the characterization of asbestos fibres. Due to problems of peak overlap in powder data, it is not possible though, to carry out a free refinement of these complex atomic structures in order to detect and measure subtle chemical changes to prove the biopersistence.

Since 2016, ID11 offered a new end station, called "nanoscope" where it is possible to focus the beam to the deep submicron scale. This is possible by using the in-line crossed silicon compound refractive lenses [3] and using a crossed pair of vertical and horizontal line foci. Combining the very small beam size with a diffractometer to align and maintain a sample in the beam during rotation is very promising for the crystallographic characterization of natural fibres. Here we report a proof of the in vivo biopersistence of asbestos fibres in human lung tissues (figure1) at the atomic scale using synchrotron micro-diffraction. We show that the atomic structure of an amosite fibre remained stable for about 40 years in the lungs of a subject diagnosed with malignant mesothelioma (MM) and originally exposed to a mixture of chrysotile, amosite and crocidolite [4].

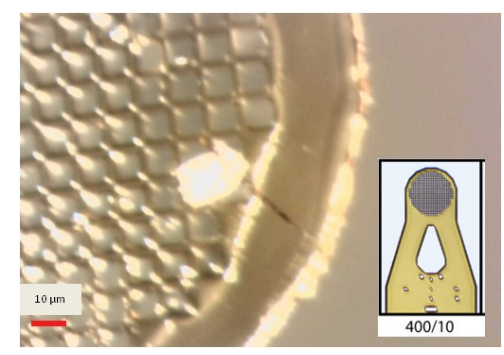

Figure 1. The amosite fibre extracted from the lung and mounted on a MiTeGen microloops ${ }^{\mathrm{TM}}$ of $400 \mu \mathrm{m}$ diameter and $10 \mu \mathrm{m}$ mesh size.

[1]Gualtieri, A.F. (2017). Introduction. In Mineral Fibres: Crystal Chemistry, Chemical-Physical Properties, Biological Interaction and Toxicity. Vol. 18. London, European Mineralogical Union-EMU Notes in Mineralogy.

[2]Manning, C. B., Vallyathan, V. \& Mossman, B. T. (2002). Int. Immunopharmacol., 2, 191-200.

[3] Snigirev, A. A., Snigireva, I., Grigoriev, M., Yunkin, V., Di Michiel, M., Kuznetsov, S. \& Vaughan, G. (2007). Proc. SPIE, 6795, 670506

[4] Giacobbe, C., Di Giuseppe, D., Zoboli,A., Lassinantti Gualtieri, M., Bonasoni,P., Moliterni, A.,Corriero, N., Altomare, A., Wright,J., \& Gualtieri, A.F. (2021). IUCrJ , 8, 76-86.

Keywords: asbestos; micro-diffraction; synchrotron. 